\title{
Genetic Diversity of Benin Cattle Populations Using Microsatellite Markers
}

\author{
Kassa Sagui Kévin ${ }^{1, ~ *}$, Dayo Guiguigbaza-Kossigan Charles ${ }^{2}$, Yapi-Gnaoré Valentine², \\ Sylla Souleymane ${ }^{2}$, Konkobo Maurice ${ }^{2}$, Youssao Abdou Karim Issaka ${ }^{1}$ \\ ${ }^{1}$ Animal Health and Production Department, Polytechnic School of Abomey-Calavi, University of Abomey-Calavi, Abomey-Calavi, Benin \\ ${ }^{2}$ International Center of Research and Development for Breeding in Subhumid Area, Bobo-Dioulasso, Burkina Faso
}

\section{Email address:}

delkassa@yahoo.fr (Kassa S. K.), charlesdayo@yahoo.fr (D. Guiguigbaza-Kossigan C.), evayapi11@yahoo.fr (Yapi-Gnaoré V.), sylsoul@yahoo.fr (S. Souleymane), mkonkobo@yahoo.fr (K. Maurice), iyoussao@yahoo.fr (Y. A. K. Issaka)

${ }^{*}$ Corresponding author

\section{To cite this article:}

Kassa Sagui Kévin, Dayo Guiguigbaza-Kossigan Charles, Yapi-Gnaoré Valentine, Sylla Souleymane, Konkobo Maurice, Youssao Abdou Karim Issaka. Genetic Diversity of Benin Cattle Populations Using Microsatellite Markers. International Journal of Animal Science and Technology. Vol. 3, No. 1, 2019, pp. 7-19. doi: 10.11648/j.ijast.20190301.12

Received: September 6, 2018; Accepted: September 19, 2018; Published: February 15, 2019

\begin{abstract}
The genetic characteristics of cattle breeds of Benin are little known. This study aimed to assess the genetic diversity of major cattle breeds of Benin. For this purpose, blood samples from 179 cattle including 31 Girolando, 30 White Fulani cattle, 31 Borgou, 31 Lagunaire, 43 Somba and 13 crossbreed Azawak x Lagunaire were characterized using 12 microsatellites markers. All used microsatellites were polymorphic within each breed of cattle. The TGLA53 marker presented the highest number of alleles (15) in the Borgou cattle while the lowest number of alleles (2) was found at the INRA 63 locus for crossbreed Azawak x Lagunaire. The observed heterozygosity rates ranged from 0.79 to 0.57 in Girolando and Somba populations respectively. The highest expected heterozygosity rates were observed in the Girolando cattle population (0.79) while the the lowest (0.63) was encountered in Lagunaire and Somba populations. The lowest value of the differentiation indice between population pairs (0.084) was observed between Borgou and White Fulani cattle. Genetic structure analysis clustered Somba and Lagunaire cattle breeds while White Fulani and Borgou cattle breeds were very close. The crossbreed populations (Borgou and Azawak x Lagunaire) were between taurine and zebu. However, taurine were introgressed $(20 \%$ on average) by the zebu blood. This study is a basic information in undertaking a conservation program and/or for support in the elaboration of a genetic improvement program for local cattle breeds of Benin.
\end{abstract}

Keywords: Borgou, White Fulani, Azawak, Girolando, Microsatellite Markers, Benin

\section{Introduction}

Benin is full of a diversity of animal genetic resources composed of cattle $(2,111,000$ head), sheep (842,000 head), goats $(1,678,000$ head $)$, pigs $(398,000$ head $)$, poultry (16,941,000 head), leporids $(25,000$ head) and grasscutters (52,000 head) [1]. From all these species, cattle provide the most important part of meat products for human consumption. These cattle are of Lagunaire, Somba, Borgou, White Fulani and Gudali breeds [2]. The main cattle of southern Benin, the Lagunaire taurine breed is rectilinear, short, ellipometric and has short tapered horns, sometimes absent with coat predominantly black, sometimes white- black [3]. The adult lagunaire weighs $142.30 \mathrm{~kg}$ [4] and produces an average of 0.36 liter of milk per day for an estimated total daily milk production of 0.42 liter [5]. Phenotypically, the Somba taurine breed is rectilinear, ellipometric and short. It has a head with small horizontal crescent-shaped horns and is distinguished by a predominantly white-black coat, black coat, or black-whited coat [6]. Its adult weighs $176 \mathrm{~kg}$ [7] and in traditional breeding, it produces an average of 0.48 liters of milk per day [6]. White Fulani cattle is rectilinear, elongated, hypermetric and has a hump and developed horns. This cattle breed usually has a white coat, gray to light gray color with black mucous membranes [8]. White Fulani adult cattle weighs 347 $\mathrm{kg}$ [4] and produces a mean of 1.45 liter of milk per day in 
traditional systems [5]. Regarding the Borgou breed, it is rectilinear, medioline and eumetric. It is characterized by a white or gray coat, sometimes white-black with gray mucous membranes and developed horns. It comes from a stabilized crossbreeding between White Fulani and Somba and incidentally Lagunaire [9]. In adulthood, the Borgou cattle weigh $288 \mathrm{~kg}$ [4] and produce a mean of 0.83 liter of milk per day with an estimate total milk of 1 liter per day in a semi-improved system [10]. In the traditional system, for a near-deep milking, the Borgou cow produces a mean of 0.99 liter of milk per day [5]. Of subconvex profile, Gudali catlle has a white color or white-red, sometimes the coat is entirely red with a hump very developed in both sexes and the horns are short or medium. It has a hypermetric format and medioline to elongated proportion [11]. The Gudali cow produces a mean of $900 \mathrm{~kg}$ of milk for a lactation period of 7 to 10 months $[12,13]$. The Gudali adult cattle weighs $449 \mathrm{~kg}$ and is characterized by a highly developed dewlap [2].

As part of the genetic characterization of these cattle in Benin, a first study was conducted by [14] on the Somba, Lagunaire, Borgou and White Fulani cattle breeds. Over the past fifteen years, the cattle landscape has been enriched with crossbreeding between local cattle breeds and the introduction of the Girolando breed in Benin to increase the level of national milk production. To do this, a new characterization of Benin cattle breeds is necessary in order to set up a program for the management and conservation of animal genetic resources and to make informed decisions on the development of these different breeds.

The purpose of this study was to:

(1) evaluate the polymorphisms of microsatellite markers in populations of White Fulani, Borgou, Somba and Lagunaire cattle breeds, as well as Girolando cattle and products derived from crosses between Azawak $\mathrm{x}$ Lagunaire cattle;

(2) determine the richness and allelic frequencies of these markers within these bovine populations;

(3) determine the indices of differentiation and the genetic structure of these cattle breeds.

\section{Materials and Methods}

\subsection{Study Area}

The blood samples for the genetic characterization of cattle breeds in Benin were collected at the farm of Samiondji for the Lagunaire and crossbred Azawak x Lagunaire cattle, at the farm of Okpara, for Borgou and Girolando cattle, in the peri-urban farms of Parakou for the White Fulani cattle and in the department of Atacora, township of Boukombe for the Somba cattle. After the collection, the blood samples were sent for DNA extraction and microsatellites genotyping to the laboratory of the International Center for Research and Development for breeding in Subhumid area (CIRDES) in the town of Bobo-Dioulasso (Burkina Faso).

\subsubsection{Livestock Farm of Samiondji}

Livestock farm of Samiondji (Figure 1) is located in department of Zou, township of Zangnanado, specifically in district of Banamè, in village of Samiondji. It is located between $2^{\circ} 22$ and $2^{\circ} 25$ East longitude, and $7^{\circ} 25$ and $7^{\circ} 30$ North latitude. The Livestock farm of Samiondji covers an area of over 3600 hectares and is totally surrounded by water. The climate is of intermediate type between the subequatorial maritime climate and the Sudano-Guinean climate, characterized by a bimodal rainfall regime with four seasons: a long dry season (November to March), a long rainy season (March to July), a short dry season (July to August) and a short rainy season (August to November). The mean rainfall varies between 900 and $1100 \mathrm{~mm}$ per year. The mean annual temperature is around $29^{\circ} \mathrm{C}$.

\subsubsection{Livestock Farm of OKPARA}

The livestock farm of Okpara (Figure 1) is located in the department of Borgou, township of Tchaourou, district of Kika ( $2^{\circ} 39-2^{\circ} 53$ longitude East and $9^{\circ} 6-9^{\circ} 21$ latitude North). It is located in the East and $15 \mathrm{~km}$ from the town of Parakou. It covers 33000 hectares of which about 10000 are actually controlled. The climate is Sudanese with alternating rainy season (May to October) and a dry season (November to April). The mean annual rainfall is $1125 \mathrm{~mm}$ and the mean annual temperature varies between 26 and $27^{\circ} \mathrm{C}$.

\subsubsection{Peri-urban Area of Parakou}

Located in the department of Borgou, the town of Parakou (Figure 1) covers $441 \mathrm{~km}^{2}$ for a density of 340 inhabitants per $\mathrm{km}^{2}$. It is located at 369 meters altitude with a latitude of $9^{\circ} 21$ North and a longitude of $2^{\circ} 37^{\prime} 0^{\prime \prime}$ East. The climate of Parakou is of Sudanese type with alternance of a rainy season (May to October) and a dry season (November to April). The annual rainfall is between 858 and $1400 \mathrm{~mm}$. The mean annual temperature varies between 26 and $27^{\circ} \mathrm{C}$.

\subsubsection{Township of Boukombe}

The township of Boukombe (Figure 1) is located in the North-West in department of Atacora. It is located between $10^{\circ}$ and $10^{\circ} 40^{\prime}$ North latitude and $0^{\circ} 75^{\prime}$ and $1^{\circ} 30$ 'East longitude. The area of Boukombe is $1,036 \mathrm{~km}^{2}$ of which 342 $\mathrm{km}^{2}$ are cultivated $(33 \%)$. The township of Boukombe is limited to the North-East by the township of Tanguiéta, to the North-West by that of Cobly, to the South by the township of Natitingou, to the East by the commune of Toucountouna and to the West by the Republic of Togo. Boukombe is in a Sudano-Guinean climate characterized by a rainy season from April to October and a dry season from November to March. The monthly temperature differences remain low $\left(36^{\circ} \mathrm{C}\right.$ in March to the warmest and $24^{\circ} \mathrm{C}$ in August to the coolest) and the mean annual rainfall is $1100 \mathrm{~mm}$. In the the township of Boukombe there is harmattan, dry and cold wind blowing between the months of November and February. 


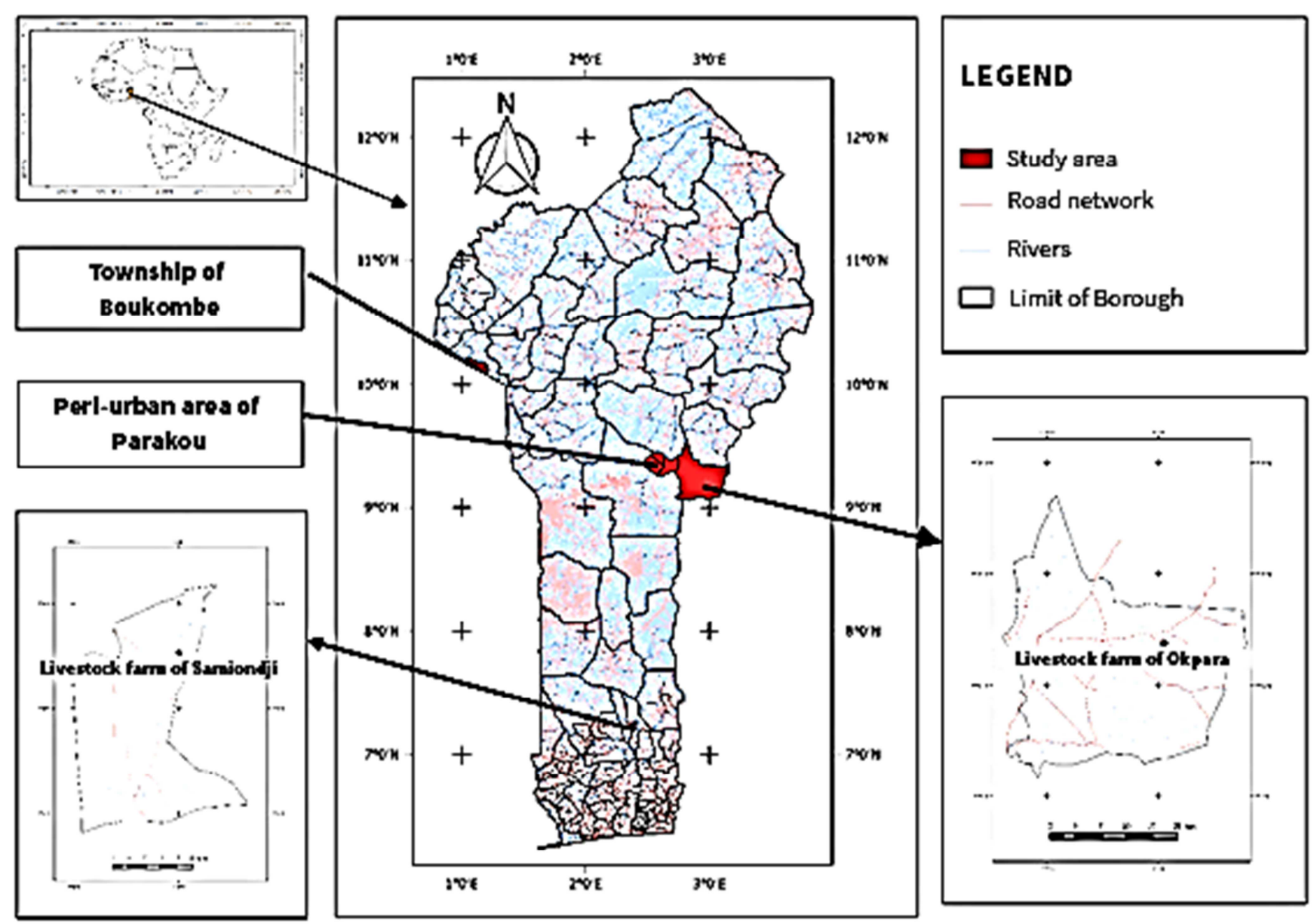

Figure 1. Map of Benin showing the different areas of the study.

Additional Legend: Indications of latitude and longitude around the map: at Left of map: $7^{\prime} 0^{\prime} \mathrm{N}, 8^{\prime} 0^{\prime} \mathrm{N}, 9^{\prime} 0^{\prime} \mathrm{N}, 10^{\prime} 0^{\prime} \mathrm{N}, 11^{\prime} 0^{\prime} \mathrm{N}, 1^{\prime} 0^{\prime} \mathrm{N}$; on right of map; $7^{\prime} 0^{\prime} \mathrm{N}, 8^{\prime} 0^{\prime} \mathrm{N}, 9^{\prime} 0^{\prime} \mathrm{N}, 10^{\prime} 0^{\prime} \mathrm{N}, 11^{\prime} 0^{\prime} \mathrm{N}, 12^{\prime} 0^{\prime} \mathrm{N}$; below of map: 1'0'E, 2'0'E, 3’0'E; above of map: 1'0'E, 2'0'E, 3'0'E

Study area: at left: Livestock farm of Samiondji; Peri-urban area of Parakou; Livestock farm of Samiondji.

On right: Livestock farm of Okpara

\subsection{Sampling Collection Procedure}

In total, blood samples from 179 cattle (31 Girolando, 30 White Fulani, 31 Borgou, 31 Lagunaire, 43 Somba and 13 Azawak x Lagunaire crossbreds) were collected from the jugular vein in vacutainer tubes containing Ethyl Diamine Tetraacetic (EDTA) as anti-coagulant. These tubes were immediately labeled (township, province, district, village, farm name, park name, cattle breed, number or name of the concerned cattle) and put in a cooler and conveyed to the CIRDES genotyping laboratory.

\subsection{DNA Amplification and Genotyping of Individuals}

DNA extraction was performed using the Promega ${ }^{\circledR}$ kit following the manufacturer's recommendations. The concentration and purity of the extracted DNAs were measured with a Spectrophotometer (NanoDrop). Twelve microsatellite markers were used for genotyping of animals. From these markers, eleven were selected from the panel of microsatellite markers established and recommended by the ISAG-FAO Consultative Group on Animal Genetic Diversity for biodiversity studies. The BM4440 microsatellite marker located on bovine chromosome 2 was chosen because it was identified as a selection signature on trypanotolerance in West African taurines [15]. The characteristics of these markers are presented in Table 1. The DNA samples were amplified by PCR in a reaction volume of $15 \mu \mathrm{l}$ using a thermocycler (BiometrTradien, version $4.20 \mathrm{~g}$, Model No.1912460, Whatman). The PCR program includes an initial phase of denaturation $\left(94^{\circ} \mathrm{C}\right.$ for 3 minutes), 35 cycles and a final phase of extension $\left(72^{\circ} \mathrm{C}\right.$ in 8 minutes). Three stages characterize each PCR cycle:

Step 1: Denaturation of both strands of DNA at high temperature in 30 seconds $\left(94^{\circ} \mathrm{C}\right)$.

Step 2: hybridization of the primers at the flanking regions of the sequence to be amplified in 30 seconds (the temperature varies between 50 and $60^{\circ} \mathrm{C}$ ) as a function of the guanine and cytosine composition and of the specificity of the oligonucleotides used as primers).

Step 3: Elongation of the neoformed strands at $72^{\circ} \mathrm{C}$ for 45 seconds.

The PCR was carried out in a reaction volume of $15 \mu \mathrm{l}$ 
including a buffer $10 \mathrm{X}$, the $1.6 \mu \mathrm{l}$ of $\mathrm{dNTP}(2.5 \mathrm{mM}), 0.8 \mu \mathrm{l}$ of $\mathrm{MgCl} 2(25 \mathrm{mM}), 0.2 \mu \mathrm{l}$ of the primer Forward with M13 $(10 \mu \mathrm{m}), 0.3 \mu \mathrm{l}$ of the reverse primer $(10 \mu \mathrm{m}), 0.1 \mu \mathrm{l}$ of the Taq polymerase $(5 \mathrm{U} / \mu \mathrm{l}), 0.3 \mu \mathrm{l}$ of dye IR 700 or $800,8.01 \mu \mathrm{l}$ of biomol water and $2 \mu$ of DNA from each sample.

The amplification products of the twelve microsatellite markers were multiplexed according to the flurochromes used (dye IR700 or dye IR800) and then denatured at $94^{\circ} \mathrm{C}$ for 3 minutes before being migrated by high voltage vertical electrophoresis (1500 volts for 1 hour 30 minutes) on a $6.5 \%$ acrylamide gel mounted on a Li-Cor (DNA Analyzer 4300) sequencer.

The detection of alleles was made using the two lasers of the sequencer Li-Cor. The analysis of the migration profiles was carried out using the SAGAGT software developed for the reading of microsatellite DNA profiles in diploid organisms.

Table 1. Characteristics of the microsatellite markers used.

\begin{tabular}{|c|c|c|c|c|c|c|c|}
\hline Microsatellite & Primers & Sequences of Primers & $\begin{array}{l}\text { Nucleotide } \\
\text { pattern }\end{array}$ & $\begin{array}{l}\text { Number of } \\
\text { chromosome }\end{array}$ & $\begin{array}{l}\text { Hybridization } \\
\text { temperature }\left({ }^{\circ} \mathrm{C}\right)\end{array}$ & $\begin{array}{l}\text { Concentratio } \\
\text { n of } \mathbf{M g C l}_{2}\end{array}$ & $\begin{array}{l}\text { Theoretical } \\
\text { size }\end{array}$ \\
\hline BM1818 & $\begin{array}{l}\text { Forward } \\
\text { Reverse }\end{array}$ & $\begin{array}{l}\text { AGCTGGGAATATAACCAAAGG } \\
\text { AGTGCTTTCAAGGTCCATGC }\end{array}$ & Di & 23 & 55 & 1.5 & $248-272$ \\
\hline BM1824 & $\begin{array}{l}\text { Forward } \\
\text { Reverse }\end{array}$ & $\begin{array}{l}\text { GAGCAAGGTGTTTTTCCCAATC } \\
\text { CATTCTCCAACTGCTTCCTTG }\end{array}$ & Di & 1 & 60 & 1.5 & $179-197$ \\
\hline BM4440 & $\begin{array}{l}\text { Forward } \\
\text { Reverse }\end{array}$ & $\begin{array}{l}\text { CCCTGGCATTCAACAAGTGT } \\
\text { TAGATGCAAAACACACACACA }\end{array}$ & Di & 2 & 60 & 1.5 & $101-129$ \\
\hline TGLA53 & $\begin{array}{l}\text { Forward } \\
\text { Reverse }\end{array}$ & $\begin{array}{l}\text { GCTTTCAGAAATAGTTTGCATTCA } \\
\text { ATCTTCACATGATATTACAGCAGA }\end{array}$ & Di & 16 & 55 & 1.5 & $143-186$ \\
\hline TGLA122 & $\begin{array}{l}\text { Forward } \\
\text { Reverse }\end{array}$ & $\begin{array}{l}\text { CССТCCTCCAGGTAAATCAGC } \\
\text { AATCCATGGCAAATAAGTACATAC }\end{array}$ & $\mathrm{Di}$ & 21 & 60 & 1.5 & $136-182$ \\
\hline ETH10 & $\begin{array}{l}\text { Forward } \\
\text { Reverse }\end{array}$ & $\begin{array}{l}\text { GTTCAGGACTGGCCCTGCTAACA } \\
\text { CCTCCAGCCCACTTTCTCTTCTC }\end{array}$ & Di & 5 & 60 & 1.5 & $207-223$ \\
\hline ETH225 & $\begin{array}{l}\text { Forward } \\
\text { Reverse }\end{array}$ & $\begin{array}{l}\text { GATCACCTTGCCACTATTTCCT } \\
\text { ACATGACAGCCAGCTGCTACT }\end{array}$ & $\mathrm{Di}$ & 9 & 55 & 1.5 & $137-159$ \\
\hline HEL1 & $\begin{array}{l}\text { Forward } \\
\text { Reverse }\end{array}$ & $\begin{array}{l}\text { CAACAGCTATTTAACAAGGA } \\
\text { AGGCTACAGTCCATGGGATT }\end{array}$ & $\mathrm{Di}$ & 15 & 50 & 1.5 & $101-119$ \\
\hline HEL9 & $\begin{array}{l}\text { Forward } \\
\text { Reverse }\end{array}$ & $\begin{array}{l}\text { CCCATTCAGTCTTCAGAGGT } \\
\text { CACATCCATGTTCTCACCAC }\end{array}$ & Di & 8 & 60 & 1.5 & $145-169$ \\
\hline INRA37 & $\begin{array}{l}\text { Forward } \\
\text { Reverse }\end{array}$ & $\begin{array}{l}\text { GATCCTGCTTATATTTAACCAC } \\
\text { AAAATTCCATGGAGAGAGAAAC }\end{array}$ & Di & 11 & 50 & 1.5 & $114-146$ \\
\hline INRA63 & $\begin{array}{l}\text { Forward } \\
\text { Reverse }\end{array}$ & $\begin{array}{l}\text { ATTTGCACAAGCTAAATCTAACC } \\
\text { AAACCACAGAAATGCTTGGAAG }\end{array}$ & Di & 18 & 52 & 1.5 & $173-185$ \\
\hline MM12 & $\begin{array}{l}\text { Forward } \\
\text { Reverse }\end{array}$ & $\begin{array}{l}\text { CAAGACAGGTGTTTCAATCT } \\
\text { ATCGACTCTGGGGATGATGT }\end{array}$ & $\mathrm{Di}$ & 9 & 55 & 1.5 & $101-145$ \\
\hline
\end{tabular}

\subsection{Statistical Analyzes}

The polymorphism rate, the mean number of alleles per locus and per population, and the allelic richness were determined using the FSTAT version 2.9.3.2 software [16]. Allelic richness has been estimated by the rarefaction method [17]. Allelic frequencies were calculated using the GENETIX software version 4.05 [18]. The observed and expected heterozygosity rates and the differentiation index (FST) were calculated using the GENETIX version 4.05 software [18]. The fixation parameter (FIS) and their significance threshold were calculated with the FSTAT version 2.9.3.2 software [16]. The FIS values were calculated using the method of [19]. The significance test of the FIS values was performed using jackknifing and bootstrapping methods [19]. The genetic structure of the different subpopulations and the global population, it was carried out using the STRUCTURE 2.3.3 software [20]. The STRUCTURE 2.3.3 software was used to estimate the likelihood of subpopulation structuring or the likelihood that individuals originate from several mixed ancestral populations, by inferring the proportion of the genome of each animal from these possible ancestral populations. Here, the number $(\mathrm{K}=2$ to 4$)$ of populations is fixed a priori. Simultaneous representation of the breeds was performed by Correspondence Factor Analysis (CFA) using GENETIX software version 4.05 [18].

\section{Results}

\subsection{Allelic Polymorphisms and Allelic Richness Per Locus in Bovine Populations}

All microsatellite markers used in genotyping were polymorphic in all populations. In all populations, the TGLA122 marker had the highest number of alleles (18 alleles) while the BM1824 locus was the least polymorphic (5 alleles). At the population level, the TGLA 53 marker showed 15 alleles in the Borgou while the INRA 63 locus was the least polymorphic with 2 alleles in the cattle population from the Azawak $x$ Lagunaire crossbreed. The lowest allelic richness (2) was also obtained in Azawak x Lagunaire crossbreed with the INRA 63 marker and the highest value (8.86) was obtained in the Borgou breed with the TGLA53 marker. The mean number of alleles per population on all the loci was $4.58,8.17,8.67$ and 6.17 respectively in the Azawak x Lagunaire, Borgou, Girolando and Lagunaire populations. The highest mean number of 
alleles (7.17 alleles) from the studied loci was obtained with Somba and White Fulani cattle populations. The number of alleles and the allelic richness per locus and per population are given in Table 2.

Table 2. Number of alleles (A) and allelic richness (AR) per locus and population.

\begin{tabular}{|c|c|c|c|c|c|c|c|}
\hline \multicolumn{2}{|c|}{ Microsatellite } & \multirow{2}{*}{$\begin{array}{l}\text { Azawak } x \\
\text { Lagunaire } \\
7\end{array}$} & \multirow{2}{*}{$\begin{array}{l}\text { Borgou } \\
7\end{array}$} & \multirow{2}{*}{$\begin{array}{l}\text { Girolando } \\
8\end{array}$} & \multirow{2}{*}{$\begin{array}{l}\text { Lagunaire } \\
7\end{array}$} & \multirow{2}{*}{$\begin{array}{l}\text { Somba } \\
9\end{array}$} & \multirow{2}{*}{$\begin{array}{l}\text { White } \\
\text { Fulani } \\
7\end{array}$} \\
\hline & $\mathrm{A}$ & & & & & & \\
\hline Div110 10 & AR & 6.57 & 5.82 & 4.9 & 5.59 & 6.27 & 5.85 \\
\hline \multirow{2}{*}{ BM1824 } & A & 4 & 4 & 5 & 4 & 3 & 5 \\
\hline & $\mathrm{AR}$ & 3.94 & 3.96 & 4.66 & 2.57 & 2.96 & 4.24 \\
\hline BM4440 & A & 6 & 7 & 11 & 6 & 5 & 7 \\
\hline \multirow{2}{*}{ ETH10 } & A & 5 & 8 & 9 & 3 & 6 & 7 \\
\hline & $\mathrm{AR}$ & 4.43 & 5.88 & 6.63 & 2.2 & 4.05 & 5.81 \\
\hline \multirow{2}{*}{ ETH225 } & A & 5 & 8 & 7 & 6 & 7 & 6 \\
\hline & AR & 4.71 & 5.35 & 5.18 & 4.54 & 5.58 & 4.67 \\
\hline \multirow{2}{*}{ HEL1 } & A & 4 & 6 & 10 & 4 & 4 & 5 \\
\hline & AR & 3.67 & 3.95 & 6.69 & 3.89 & 3.07 & 4.09 \\
\hline HEL9 & A & 4 & 9 & 11 & 6 & 8 & 9 \\
\hline \multirow{2}{*}{ INRA63 } & A & 2 & 5 & 6 & 6 & 6 & 3 \\
\hline & $\mathrm{AR}$ & 2 & 3.83 & 4.24 & 4.66 & 3.99 & 2.55 \\
\hline \multirow{2}{*}{ INRA37 } & A & 4 & 9 & 7 & 7 & 9 & 7 \\
\hline & AR & 4 & 6.76 & 5.76 & 5.45 & 5.75 & 5.36 \\
\hline \multirow{2}{*}{ MM12 } & A & 5 & 9 & 8 & 6 & 8 & 9 \\
\hline & $\mathrm{AR}$ & 4.71 & 5.91 & 5.06 & 4.01 & 4.4 & 6.90 \\
\hline \multirow{2}{*}{ TGLA122 } & A & 5 & 11 & 9 & 7 & 8 & 10 \\
\hline & AR & 5 & 5.92 & 6.49 & 5.31 & 5.82 & 4.99 \\
\hline \multirow{2}{*}{ TGLA53 } & A & 4 & 15 & 13 & 12 & 13 & 11 \\
\hline & AR & 3.97 & 8.86 & 7.46 & 6.44 & 6.69 & 7.12 \\
\hline \multicolumn{2}{|c|}{ Mean number of alleles per population across all loci } & 4.58 & 8.17 & 8.67 & 6.17 & 7.17 & 7.17 \\
\hline \multicolumn{2}{|c|}{ Mean allelic wealth per population across all loci } & 4.31 & 5.60 & 6.03 & 4.44 & 4.69 & 5.20 \\
\hline
\end{tabular}

\subsection{Distribution of Allelic Frequencies of Microsatellite Loci in Bovine Populations}

Alleles at microsatellite loci are unevenly distributed among different populations. Some alleles have presented high frequencies in some populations. Thus, the allele 119 of the
BM4440 locus characterized the Somba, Borgou and Lagunaire populations with allelic frequencies of 0.90 .0 .75 and 0.67 respectively. Likewise, the allele 201 of locus BM 1824 had high frequencies of 0.78 and 0.58 respectively in the Lagunaire and Somba populations. Tables 3 to 14 present the frequencies of the alleles at the different loci in the 6 cattle populations.

Table 3. Distribution of allelic frequencies at BM1818 locus.

\begin{tabular}{|c|c|c|c|c|c|c|}
\hline Alleles & Azawak x Lagunaire & Borgou & Girolando & Lagunaire & Somba & White Fulani \\
\hline 274 & 0.05 & 0.03 & 0 & 0.1 & 0.1 & 0.12 \\
\hline 276 & 0.23 & 0.05 & 0.1 & 0.4 & 0.23 & 0 \\
\hline 278 & 0 & 0.19 & 0.1 & 0.1 & 0.15 & 0.12 \\
\hline 282 & 0.09 & 0 & 0 & 0 & 0.1 & 0 \\
\hline 284 & 0.14 & 0.19 & 0.5 & 0.1 & 0.1 & 0.05 \\
\hline 286 & 0.14 & 0.16 & 0 & 0 & 0.13 & 0.2 \\
\hline 290 & 0.27 & 0.1 & 0 & 0 & 0.01 & 0.22 \\
\hline 292 & 0 & 0 & 0 & 0 & 0.01 & 0 \\
\hline 298 & 0 & 0 & 0 & 0 & 0 & 0 \\
\hline
\end{tabular}

Table 4. Distribution of allelic frequencies at BM1824 locus.

\begin{tabular}{lllllll}
\hline Alleles & Azawak x Lagunaire & Borgou & Girolando & Lagunaire & Somba \\
\hline 199 & 0.15 & 0.23 & 0.26 & 0.02 & 0.24 & 0.29 \\
201 & 0.46 & 0.36 & 0.28 & 0.78 & 0.58 \\
203 & 0.27 & 0.25 & 0.28 & 0.19 & 0.18 \\
207 & 0 & 0 & 0.09 & 0 & 0 \\
211 & 0.12 & 0.16 & 0.09 & 0.02 & 0.16 \\
\hline
\end{tabular}


Table 5. Distribution of allelic frequencies at BM4440 locus.

\begin{tabular}{|c|c|c|c|c|c|c|}
\hline Alleles & Azawak x Lagunaire & Borgou & Girolando & Lagunaire & Somba & White Fulani \\
\hline 117 & 0 & 0.04 & 0 & 0 & 0 & 0.02 \\
\hline 119 & 0.29 & 0.75 & 0.45 & 0.67 & 0.9 & 0.66 \\
\hline 121 & 0.04 & 0.08 & 0.07 & 0.04 & 0.04 & 0.04 \\
\hline 123 & 0 & 0 & 0.02 & 0 & 0 & 0 \\
\hline 125 & 0 & 0.02 & 0 & 0 & 0 & 0 \\
\hline 127 & 0 & 0 & 0.02 & 0 & 0 & 0.02 \\
\hline 129 & 0.33 & 0 & 0.12 & 0.17 & 0.01 & 0.04 \\
\hline 133 & 0 & 0 & 0.02 & 0 & 0 & 0 \\
\hline 135 & 0 & 0 & 0.07 & 0 & 0 & 0 \\
\hline 139 & 0 & 0.04 & 0.07 & 0.06 & 0.02 & 0.18 \\
\hline 141 & 0.17 & 0.04 & 0.05 & 0.04 & 0 & 0.04 \\
\hline 143 & 0.04 & 0.04 & 0 & 0.02 & 0.02 & 0.02 \\
\hline 145 & 0 & 0 & 0.07 & 0 & 0 & 0 \\
\hline
\end{tabular}

Table 6. Distribution of allelic frequencies at ETH1O locus.

\begin{tabular}{lllllll}
\hline Alleles & Azawak x Lagunaire & Borgou & Girolando & Lagunaire & Somba & White Fulani \\
\hline 223 & 0 & 0.03 & 0.02 & 0 & 0 \\
225 & 0.08 & 0.27 & 0.08 & 0 & 0.03 \\
227 & 0.38 & 0.07 & 0.08 & 0 & 0.2 \\
229 & 0.04 & 0.22 & 0.15 & 0.02 & 0 \\
231 & 0 & 0.02 & 0.06 & 0 & 0 \\
233 & 0 & 0.07 & 0 & 0 & 0.11 \\
235 & 0 & 0.07 & 0 & 0 & 0.09 \\
237 & 0.38 & 0.27 & 0.02 & 0.85 & 0.03 \\
239 & 0.12 & 0 & 0.11 & 0.13 & 0.07 \\
\hline
\end{tabular}

Table 7. Distribution of allelic frequencies at ETH 225 locus.

\begin{tabular}{llllll}
\hline Alleles & Azawak x Lagunaire & Borgou & Girolando & Lagunaire & Somba \\
\hline 158 & 0.15 & 0.24 & 0.05 & 0.15 & 0.41 \\
160 & 0 & 0 & 0 & 0 & 0.05 \\
162 & 0 & 0.03 & 0.02 & 0.11 & 0.22 \\
164 & 0.08 & 0.29 & 0.23 & 0.48 & 0 \\
166 & 0.23 & 0.08 & 0.1 & 0.21 & 0.22 \\
168 & 0 & 0.05 & 0.18 & 0 & 0.11 \\
170 & 0.08 & 0.02 & 0.03 & 0.02 & 0.04 \\
172 & 0 & 0 & 0 & 0 & 0 \\
174 & 0.46 & 0.27 & 0.38 & 0.03 & 0.03 \\
176 & 0 & 0.02 & 0 & 0 & 0.07 \\
\hline
\end{tabular}

Table 8. Distribution of allelic frequencies at HEL1 locus.

\begin{tabular}{llllll}
\hline Alleles & Azawak x Lagunaire & Borgou & Girolando & Lagunaire & Somba \\
\hline 119 & 0 & 0.02 & 0 & 0 & 0 \\
121 & 0.29 & 0.42 & 0.02 & 0.52 & 0 \\
123 & 0.04 & 0.05 & 0.07 & 0.15 & 0.73 \\
125 & 0.46 & 0.42 & 0.31 & 0.17 & 0.18 \\
129 & 0.21 & 0.08 & 0.02 & 0.15 & 0.03 \\
131 & 0 & 0 & 0.07 & 0 & 0.06 \\
133 & 0 & 0 & 0.09 & 0 & 0 \\
135 & 0 & 0 & 0.26 & 0 & 0 \\
139 & 0 & 0.02 & 0.07 & 0 & 0 \\
\hline
\end{tabular}

Table 9. Distribution of allelic frequencies at HEL 9 locus.

\begin{tabular}{lllllll}
\hline Alleles & Azawak x Lagunaire & Borgou & Girolando & Lagunaire & Somba & White Fulani \\
\hline 168 & 0 & 0.02 & 0.05 & 0 & 0 \\
170 & 0 & 0 & 0.07 & 0 & 0 \\
172 & 0 & 0.11 & 0.09 & 0.02 & 0 \\
174 & 0.05 & 0.14 & 0.03 & 0.07 & 0.12 \\
176 & 0 & 0.13 & 0.24 & 0 & 0.08 \\
178 & 0 & 0.04 & 0.03 & 0 & 0.01 \\
180 & 0.59 & 0.29 & 0.17 & 0.46 & 0.1 \\
182 & 0 & 0.07 & 0.1 & 0.22 & 0.46 \\
\hline
\end{tabular}




\begin{tabular}{lllllll}
\hline Alleles & Azawak x Lagunaire & Borgou & Girolando & Lagunaire & Somba & White Fulani \\
\hline 184 & 0.32 & 0.18 & 0.07 & 0.19 & 0.07 & 0.09 \\
186 & 0.05 & 0 & 0.07 & 0 & 0.01 & 0.02 \\
188 & 0 & 0.04 & 0.07 & 0 & 0.05 & 0.28 \\
190 & 0 & 0 & 0 & 0.04 & 0 & 0 \\
\hline
\end{tabular}

Table 10. Distribution of allelic frequencies at INRA 63 locus.

\begin{tabular}{lllllll}
\hline Alleles & Azawak x Lagunaire & Borgou & Girolando & Lagunaire & Somba & White Fulani \\
\hline 192 & 0 & 0 & 0 & 0.04 & 0.01 & 0 \\
194 & 0 & 0.02 & 0.22 & 0.06 & 0.09 & 0 \\
196 & 0.58 & 0.23 & 0.4 & 0.2 & 0.34 & 0.06 \\
198 & 0 & 0 & 0.02 & 0 & 0.04 & 0 \\
200 & 0 & 0.19 & 0.02 & 0.04 & 0.04 & 0.11 \\
202 & 0.42 & 0.52 & 0.28 & 0.35 & 0.49 & 0.83 \\
204 & 0 & 0.05 & 0.07 & 0.31 & 0 & 0 \\
\hline
\end{tabular}

Table 11. Distribution of allelic frequencies at INRA 37 locus.

\begin{tabular}{lllllll}
\hline Alleles & Azawak x Lagunaire & Borgou & Girolando & Lagunaire & Somba & White Fulani \\
\hline 132 & 0 & 0 & 0 & 0.09 & 0 & 0 \\
138 & 0 & 0.07 & 0 & 0 & 0.06 & 0 \\
140 & 0 & 0.02 & 0.23 & 0 & 0 & 0.06 \\
142 & 0 & 0.07 & 0.03 & 0.02 & 0.04 & 0.07 \\
144 & 0 & 0.13 & 0.03 & 0.32 & 0.13 & 0.38 \\
146 & 0.25 & 0.37 & 0.07 & 0.27 & 0.54 & 0.14 \\
148 & 0.56 & 0.13 & 0.2 & 0.16 & 0.04 & 0.29 \\
150 & 0.06 & 0.09 & 0.1 & 0.02 & 0.11 & 0.05 \\
152 & 0.13 & 0.11 & 0.33 & 0.11 & 0.02 & 0 \\
154 & 0 & 0.02 & 0 & 0 & 0.02 & 0 \\
158 & 0 & 0 & 0 & &
\end{tabular}

Table 12. Distribution of allelic frequencies at MM12 locus.

\begin{tabular}{|c|c|c|c|c|c|c|}
\hline Alleles & Azawak x Lagunaire & Borgou & Girolando & Lagunaire & Somba & White Fulan \\
\hline 121 & 0 & 0 & 0.06 & 0.02 & 0 & 0.03 \\
\hline 127 & 0.08 & 0.08 & 0.02 & 0 & 0 & 0.17 \\
\hline 135 & 0.15 & 0.1 & 0 & 0 & 0 & 0 \\
\hline 137 & 0 & 0 & 0.05 & 0.06 & 0.03 & 0.17 \\
\hline 139 & 0.42 & 0.45 & 0 & 0.56 & 0.47 & 0.2 \\
\hline 141 & 0.27 & 0.16 & 0.08 & 0.26 & 0.34 & 0.10 \\
\hline 143 & 0 & 0.03 & 0.34 & 0.02 & 0.01 & 0.18 \\
\hline 145 & 0 & 0 & 0.03 & 0 & 0 & 0.08 \\
\hline 147 & 0 & 0 & 0 & 0 & 0.01 & 0.02 \\
\hline 151 & 0 & 0.03 & 0.02 & 0 & 0.01 & 0 \\
\hline 153 & 0 & 0.02 & 0.4 & 0 & 0 & 0 \\
\hline 157 & 0.08 & 0.11 & 0 & 0.08 & 0.06 & 0.05 \\
\hline 165 & 0 & 0.02 & 0 & 0 & 0.07 & 0 \\
\hline
\end{tabular}

Table 13. Distribution of allelic frequencies at TGLA122 locus.

\begin{tabular}{|c|c|c|c|c|c|c|}
\hline Alleles & Azawak x Lagunaire & Borgou & Girolando & Lagunaire & Somba & White Fulan \\
\hline 156 & 0.06 & 0.07 & 0.18 & 0 & 0.02 & 0.05 \\
\hline 158 & 0 & 0.02 & 0 & 0.1 & 0.09 & 0 \\
\hline 160 & 0 & 0 & 0 & 0 & 0.02 & 0 \\
\hline 162 & 0.63 & 0.48 & 0.29 & 0.18 & 0.43 & 0.58 \\
\hline 164 & 0.13 & 0.2 & 0.13 & 0.05 & 0.09 & 0.18 \\
\hline 166 & 0 & 0.02 & 0.02 & 0.23 & 0.07 & 0.02 \\
\hline 168 & 0 & 0 & 0.16 & 0 & 0 & 0 \\
\hline 170 & 0.06 & 0.08 & 0.13 & 0.4 & 0.22 & 0.08 \\
\hline 172 & 0 & 0.03 & 0.05 & 0 & 0.07 & 0.02 \\
\hline 176 & 0 & 0 & 0 & 0.03 & 0 & 0 \\
\hline 178 & 0 & 0 & 0 & 0.03 & 0 & 0.02 \\
\hline 180 & 0.13 & 0.05 & 0 & 0 & 0 & 0 \\
\hline 182 & 0 & 0 & 0 & 0 & 0 & 0.02 \\
\hline 184 & 0 & 0.02 & 0 & 0 & 0 & 0 \\
\hline 186 & 0 & 0.02 & 0 & 0 & 0 & 0 \\
\hline 188 & 0 & 0 & 0.02 & 0 & 0 & 0 \\
\hline
\end{tabular}




\begin{tabular}{llllll}
\hline Alleles & Azawak x Lagunaire & Borgou & Girolando & Lagunaire & Somba \\
\hline 190 & 0 & 0.02 & 0 & 0 & 0 \\
194 & 0 & 0.02 & 0 & 0 & 0.02 \\
\hline
\end{tabular}

Table 14. Distribution of allelic frequencies at TGLA53 locus.

\begin{tabular}{|c|c|c|c|c|c|c|}
\hline Alleles & Azawak x Lagunaire & Borgou & Girolando & Lagunaire & Somba & White Fulani \\
\hline 172 & 0 & 0.02 & 0.02 & 0.04 & 0.04 & 0 \\
\hline 174 & 0 & 0.06 & 0 & 0.02 & 0.21 & 0.02 \\
\hline 176 & 0 & 0 & 0 & 0 & 0.01 & 0 \\
\hline 178 & 0.29 & 0.21 & 0.36 & 0.11 & 0 & 0.28 \\
\hline 180 & 0.21 & 0 & 0.07 & 0.02 & 0.03 & 0 \\
\hline 182 & 0 & 0.06 & 0.04 & 0 & 0.05 & 0 \\
\hline 186 & 0.38 & 0.02 & 0 & 0.02 & 0.1 & 0.25 \\
\hline 188 & 0 & 0.06 & 0.05 & 0.04 & 0.13 & 0.05 \\
\hline 190 & 0 & 0.06 & 0.02 & 0.05 & 0.04 & 0.08 \\
\hline 192 & 0.13 & 0.02 & 0 & 0.02 & 0.01 & 0.03 \\
\hline 194 & 0 & 0.04 & 0.02 & 0.02 & 0.01 & 0.05 \\
\hline 196 & 0 & 0.06 & 0 & 0 & 0 & 0.12 \\
\hline 198 & 0 & 0.02 & 0.18 & 0.07 & 0 & 0 \\
\hline 202 & 0 & 0.12 & 0.05 & & 0.01 & 0.03 \\
\hline 204 & 0 & 0.04 & 0.02 & 0 & 0.01 & 0.07 \\
\hline
\end{tabular}

\subsection{Observed and Expected Heterozygosity}

Observed heterozygosities in the studied cattle populations ranged from 0.57 for the Somba breed to 0.79 for the Girolando. Expected heterozygosity ranged from 0.63 in Lagunaire and Somba cattle to 0.79 Girolando's population. The different values of the heterozygosity rates are shown in Table 15.

Table 15. Expected $(E H)$ and observed $(\mathrm{OH})$ heterozygosity values by locus and their mean values per population.

\begin{tabular}{|c|c|c|c|c|c|c|c|}
\hline Loci & Azawak x Lagunaire & Borgou & Girolando & Lagunaire & Somba & White Fulani & Mean \\
\hline \multicolumn{8}{|l|}{ BM1818 } \\
\hline$(\mathrm{N})$ & 11 & 31 & 30 & 25 & 41 & 30 & \\
\hline $\mathrm{EH}$ & 0.86 & 0.82 & 0.67 & 0.77 & 0.84 & 0.82 & 0.8 \\
\hline $\mathrm{OH}$ & 1 & 0.81 & 0.57 & 0.8 & 0.9 & 0.87 & 0.82 \\
\hline \multicolumn{8}{|l|}{ BM1824 } \\
\hline$(\mathrm{N})$ & 13 & 22 & 23 & 27 & 44 & 28 & \\
\hline $\mathrm{EH}$ & 0.7 & 0.75 & 0.77 & 0.37 & 0.58 & 0.76 & 0.65 \\
\hline $\mathrm{OH}$ & 0.54 & 0.77 & 0.74 & 0.41 & 0.57 & 0.86 & 0.65 \\
\hline \multicolumn{8}{|l|}{ BM4440 } \\
\hline$(\mathrm{N})$ & 12 & 26 & 21 & 26 & 42 & 25 & \\
\hline $\mathrm{EH}$ & 0.79 & 0.43 & 0.77 & 0.52 & 0.18 & 0.54 & 0.54 \\
\hline $\mathrm{OH}$ & 0.92 & 0.38 & 0.76 & 0.58 & 0.19 & 0.52 & 0.56 \\
\hline \multicolumn{8}{|l|}{ ETH10 } \\
\hline$(\mathrm{N})$ & 13 & 30 & 31 & 30 & 44 & 30 & \\
\hline $\mathrm{EH}$ & 0.71 & 0.81 & 0.84 & 0.26 & 0.51 & 0.81 & 0.66 \\
\hline $\mathrm{OH}$ & 0.85 & 0.77 & 0.87 & 0.3 & 0.5 & 0.77 & 0.68 \\
\hline \multicolumn{8}{|l|}{ ETH225 } \\
\hline$(\mathrm{N})$ & 13 & 31 & 30 & 31 & 38 & 29 & \\
\hline $\mathrm{EH}$ & 0.73 & 0.78 & 0.76 & 0.7 & 0.76 & 0.73 & 0.74 \\
\hline $\mathrm{OH}$ & 0.69 & 0.77 & 0.9 & 0.61 & 0.71 & 0.69 & 0.73 \\
\hline \multicolumn{8}{|l|}{ HEL1 } \\
\hline (N) & 12 & 30 & 27 & 26 & 44 & 29 & \\
\hline EH & 0.69 & 0.65 & 0.82 & 0.67 & 0.44 & 0.66 & 0.65 \\
\hline $\mathrm{OH}$ & 0.58 & 0.57 & 0.85 & 0.69 & 0.32 & 0.76 & 0.63 \\
\hline \multicolumn{8}{|l|}{ HEL9 } \\
\hline$(\mathrm{N})$ & 11 & 28 & 29 & 27 & 42 & 29 & \\
\hline $\mathrm{EH}$ & 0.57 & 0.85 & 0.89 & 0.71 & 0.73 & 0.84 & 0.76 \\
\hline $\mathrm{OH}$ & 0.55 & 0.89 & 0.93 & 0.56 & 0.71 & 0.93 & 0.76 \\
\hline \multicolumn{8}{|l|}{ INRA63 } \\
\hline$(\mathrm{N})$ & 12 & 31 & 30 & 27 & 40 & 27 & \\
\hline $\mathrm{EH}$ & 0.51 & 0.65 & 0.72 & 0.74 & 0.65 & 0.3 & 0.59 \\
\hline $\mathrm{OH}$ & 0.83 & 0.77 & 0.9 & 0.74 & 0.55 & 0.33 & 0.69 \\
\hline
\end{tabular}




\begin{tabular}{|c|c|c|c|c|c|c|c|}
\hline Loci & Azawak x Lagunaire & Borgou & Girolando & Lagunaire & Somba & White Fulani & Mean \\
\hline \multicolumn{8}{|l|}{ INRA37 } \\
\hline$(\mathrm{N})$ & 8 & 23 & 15 & 22 & 27 & 21 & \\
\hline $\mathrm{EH}$ & 0.64 & 0.82 & 0.8 & 0.79 & 0.69 & 0.76 & 0.75 \\
\hline $\mathrm{OH}$ & 0.38 & 0.52 & 0.6 & 0.27 & 0.67 & 0.67 & 0.52 \\
\hline \multicolumn{8}{|l|}{ MM12 } \\
\hline$(\mathrm{N})$ & 13 & 31 & 31 & 31 & 43 & 30 & \\
\hline EH & 0.74 & 0.75 & 0.72 & 0.61 & 0.67 & 0.86 & 0.73 \\
\hline $\mathrm{OH}$ & 0.69 & 0.65 & 0.61 & 0.68 & 0.58 & 0.97 & 0.7 \\
\hline \multicolumn{8}{|l|}{ TGLA122 } \\
\hline$(\mathrm{N})$ & 8 & 30 & 28 & 20 & 23 & 30 & \\
\hline $\mathrm{EH}$ & 0.61 & 0.72 & 0.84 & 0.76 & 0.76 & 0.63 & 0.72 \\
\hline $\mathrm{OH}$ & 0.63 & 0.63 & 0.89 & 0.7 & 0.57 & 0.63 & 0.67 \\
\hline \multicolumn{8}{|l|}{ TGLA53 } \\
\hline$(\mathrm{N})$ & 12 & 26 & 28 & 28 & 40 & 30 & \\
\hline $\mathrm{EH}$ & 0.75 & 0.89 & 0.83 & 0.7 & 0.81 & 0.84 & 0.8 \\
\hline $\mathrm{OH}$ & 0.75 & 0.96 & 0.86 & 0.68 & 0.58 & 0.83 & 0.78 \\
\hline EH Mean & 0.69 & 0.74 & 0.79 & 0.63 & 0.63 & 0.71 & 0.7 \\
\hline OH Mean & 0.7 & 0.71 & 0.79 & 0.58 & 0.57 & 0.74 & 0.68 \\
\hline
\end{tabular}

\subsection{Differentiation Measures of the Studied Populations}

The population differentiations were calculated within and between populations and represented by the values of the individual-sub-population fixation and differentiation between population pairs indices. A significant difference $(\mathrm{p}<0.05)$ at the Hardy-Weinberg equilibrium was observed in the Borgou, Lagunaire and Somba cattle populations (Table
16). The highest values of the FST differentiation indices between the populations pairs were observed between Azawak x Lagunaire crossbreed and Girolando (0.406), Girolando and Lagunaire cattle (0.669), Girolando and Somba cattle (0.569), Lagunaire and White Fulani cattle $(0.573)$ and between Somba and White Fulani cattle (0.427). The lowest value (0.084) of FST was observed between the Borgou cattle breed and White Fulani (Table 17).

Table 16. Values of individual-sub-population fixation indices per locus and per population.

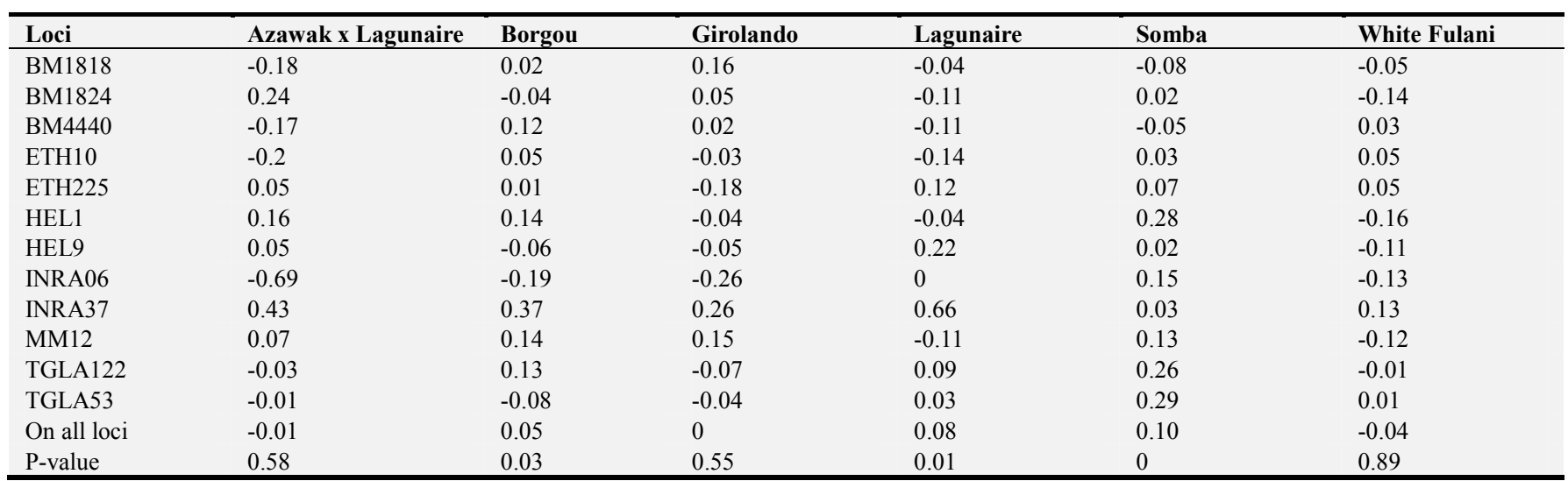

Table 17. Values of differentiation indices between population pairs.

\begin{tabular}{llllll}
\hline & Borgou & Girolando & Lagunaire & Somba & White Fulani \\
\hline Azawak x Lagunaire & 0.197 & 0.406 & 0.266 & 0.237 & 0.323 \\
Borgou & & 0.323 & 0.257 & 0.084 & 0.569 \\
Girolando & & 0.669 & 0.099 & 0.37 \\
Lagunaire & & & 0.573 \\
Somba & & & 0.427 \\
\hline
\end{tabular}

\subsection{Genetic Structure of the Studied Populations}

The factorial analysis of the correspondences of the studied cattle populations (AFC) clearly distinguished the populations of Somba and Lagunaire from White Fulani (Figure 2). The grouping of Borgou and Azawak x Lagunaire populations is intermediate between Somba. Lagunaire and White Fulani populations and is closer to White Fulani.
Girolando population differs widely from all other populations. The analysis of the genetic structure at $\mathrm{K}=2$ separates White Fulani and Girolando from crossbreed populations (Figure 3). By redefining the 6 populations at $\mathrm{K}=3$, bulls remained genetically close. It is the same for the group of populations from crossing bulls and Zebu (Borgou and Azawak x Lagunaire). On the other hand, the Girolando distinguished themselves from White Fulani cattle. At $\mathrm{K}=4$, 
we distinguished the group of bulls (Somba and Lagunaire), the group 3 consisting only of Girolando and the group 4 the group consisting of Borgou cattle and White Fulani cattle, consisting of crossbreed (Azawak x Lagunaire).

\section{bv_benin.gtx}

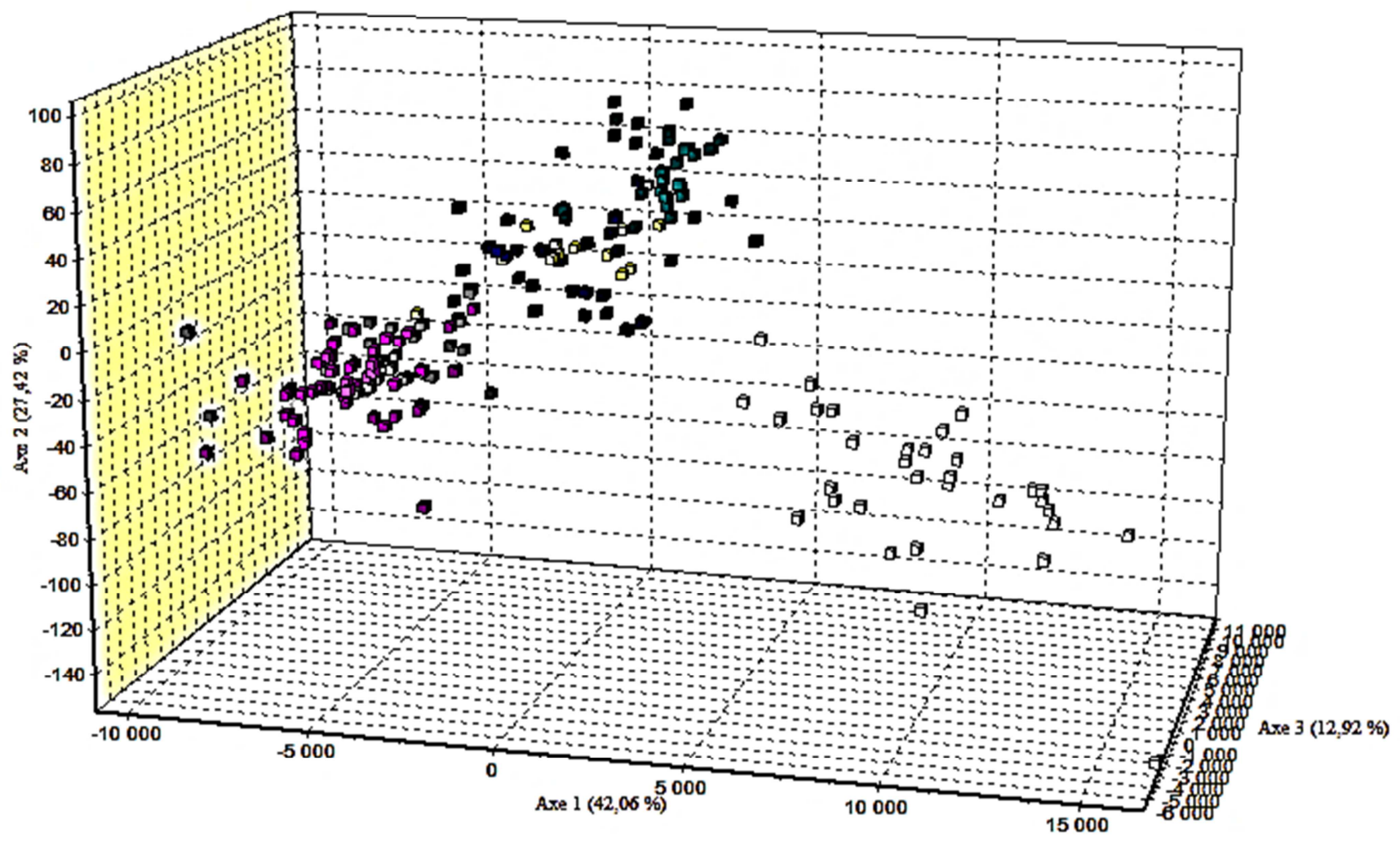

Figure 2. Factorial analysis of the correspondences of the cattle populations studied.

Girolando: White. Somba: red. Lagunaire: grey. Azawak x Lagunaire: yellow. Borgou: blue. White Fulani: green.

Below on right: Axe $3:-6000 ;-5000 ;-4000 ;-3000 ;-2000 ;-1000 ; 0 ; 1000 ; 2000 ; 3000 ; 4000 ; 5000 ; 6000 ; 7000 ; 8000 ; 9000 ; 10000 ; 11000$.
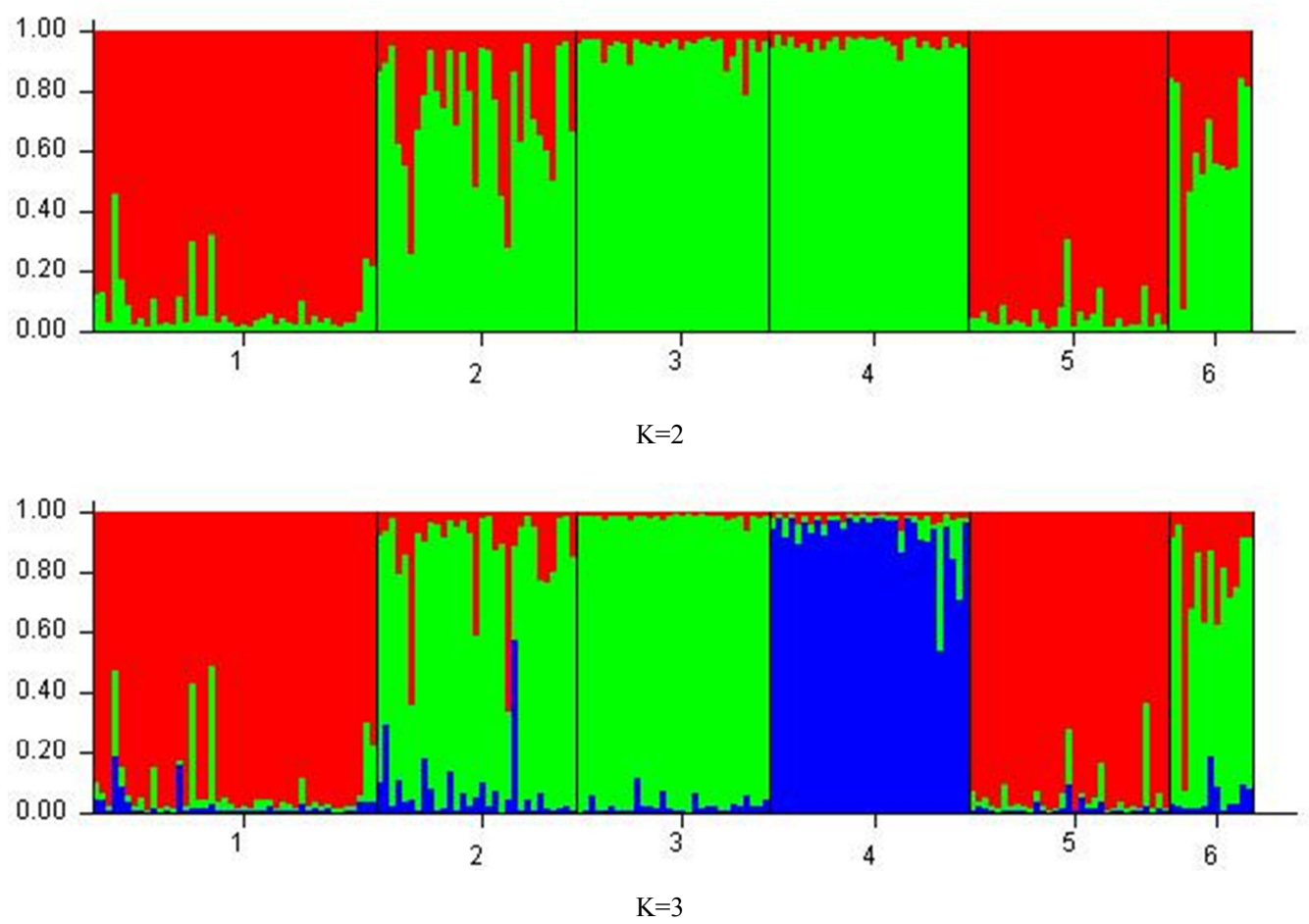


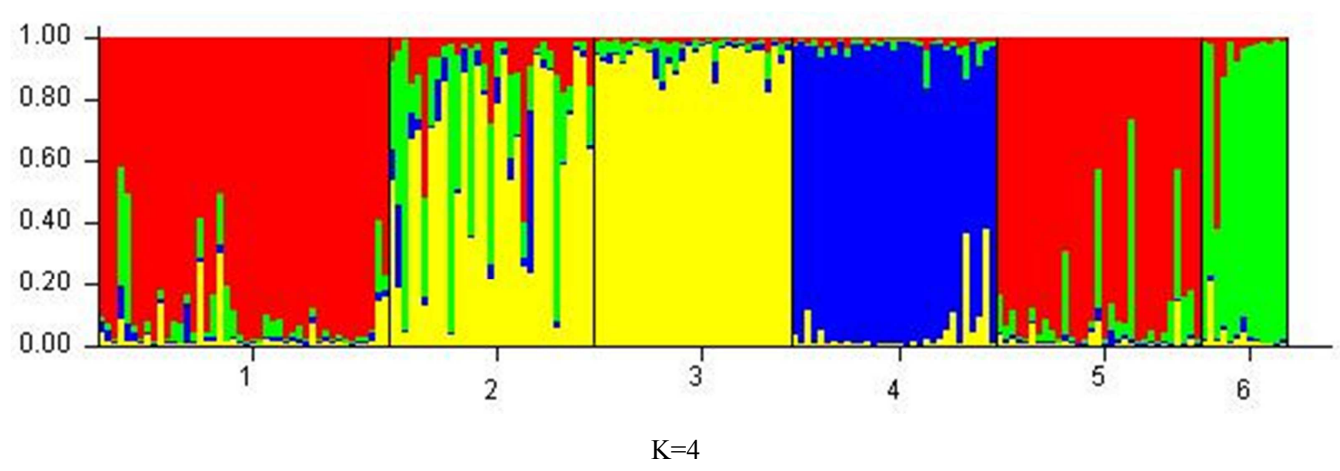

1: Somba, 2: Borgou, 3: White Fulani, 4: Girolando, 5: Lagunaire, 6: Azawak x Lagunaire

Figure 3. Genetic structure of the 6 breeds of cattle supposing $K=2,3$ and 4 .

\section{Discussion}

\subsection{Genetic Diversity of Benin Cattle Populations}

The present study showed that there is a high genetic diversity characterized by the large polymorphisms at the studied microsatellite loci, the number of alleles per locus and per population, the allelic richness and the distribution of allelic frequencies in the 6 cattle populations. The high allelic diversity observed in the Borgou and Girolando breeds compared to other breeds in this study can be explained in part by the origin of these two breeds. Indeed, the Borgou cattle would come from a stabilized distant crossbreeding between the White Fulani and the Somba cattle and incidentally Lagunaire [2]. The Girolando cattle inherit their genetic from a crossbreeding between the Gir Zebu of India and the European Holstein breed. The results of this study are comparable to those of [14], who found a mean number of allele of 6.5 in Somba and 7.9 in White Fulani. In contrast to this study, these authors found a mean of 4.5 alleles per locus in the Borgou cattle population. The sampling method of the genotyped individuals and the size of the sample can explain this difference. Indeed. [14] conducted their study on animals from five breeders in the department of Borgou while our study was carried out on cattle of the farm of Okpara.

The Okpara Breeding Farm exploits several male of Borgou breed in the herd while in extensive breeding, breeders usually use only one Borgou male parent in the herd. Sample size may be a factor in the number of alleles to different markers and it is obvious that the larger the sample size, the greater the number of alleles.

However, the sample size is smaller than that of [14]. The breeding system would then be the only factor justifying the large number of alleles observed in the population of Borgou in our study. The mean number of alleles (6.17) from Lagunaire breed is higher than that (4.7) found by [14] in this breed. This difference in alleles between the bulls of our study and those of [14] can be explained by the degree of mixing of these individuals.

Indeed, these bulls are more and more introgressed by zebu blood. This hypothesis of introgression of Benin bulls can be reinforced by the results obtained in the work of [15]. These authors had shown that contrary to what we observed in zebu, the BM4440 locus has a reduced variability in West African bulls populations resulting in the presence from 1 to 2 maximum alleles among which the $119 \mathrm{bp}$ allele (101bp in their study) had an allelic frequency higher than $95 \%$ except in the Lagunaire that presented two almost equal frequency alleles. The high number of alleles at microsatellite loci in West African bulls has also been reported by [21] in Burkina Faso. These authors reported a mean number of alleles of 6.7 per locus in N'dama bulls. It is also observed significantly lower allelic diversity in West African bulls in recent years $[22,21]$. This suggests that effective actions need to be implemented to characterize and inventory animal genetic resources for their sustainable improvement and conservation.

\subsection{Heterozygosity and Fixation Index}

The observed heterozygosity values in the present study are relatively high among crossbreeds, Borgou and Girolando cattle and can be explained by the fact that these cattle benefit from a heterogeneity coming from two genitors of different breeds among which we find zebus which are genetically heterogeneous. In fact, the Azawak x Lagunaire crossbreed has in association the alleles of Lagunaire bulls and those of Azawak Zebu. the Borgou cattle (far stabilization) are endowed with the genes of the Somba bulls and incidentally Lagunaire in addition to those of White Fulani while Girolando cattle is a product of a distant crossbreeding between Gir Zebu and Holstein cattle.

The obtained heterozygotes are near the values reported by [14] in Somba, Lagunaire, White Fulani and Borgou cattle. Similar values of heterozygosity were obtained by [21] in zebus (White Fulani, Azawak and Gudali), Lobi and N'dama bulls from Burkina Faso. The BM1818, BM1824, ETH10, ETH225 and TGLA122 microsatellite loci revealed mean values of 0.66 (Lobi), 0.7 (zebu) and 0.68 (N'dama) [21]. Overall, the fixation parameters show that there is a loss of alleles in the Borgou, Somba and Lagunaire cattle populations. The practice of selection within Borgou breed at Okpara Breeding Farm (OTF) could contribute to the fixation of some regions of the genome of individuals. Generally, for this breed, there is a preference of the white coat color with black extremities and the breeders further exploit this breed more performant by keeping the white color of the coat. On 
the other hand, herds of Somba and Lagunaire cattle breeds face the problem of male spawning renewal, which leads to consanguinity and depletion of genetic material. The population of White Fulani has a tendency to gain alleles (FIS $=-0.04$ ) and this could be explained by the migration phenomenon of genes from other cattle breeds during the transhumance period of Fulani pastoralists. Imported Girolando cattle are in Hardy Weinberg equilibrium. This population in semi-improved production system at Okpara breeding farm is rigorously followed in its reproduction. Deviations from the Hardy-Weinberg equilibrium had already been reported by [14] in the White Fulani, Borgou Lagunaire and Somba breeds. [23] also observed an allelic deficiency in Lagunaire in a study conducted in the Ouémé Valley in Benin. Unlike the present work, [24] found in White Fulani cattle from Nigeria a FIS value of 0.01 using the BM1818, BM1824, ETH10, HEL1, INRA37 and TGLA122 loci. This difference could be explained by the sampling method but also by the fact that the two studies were carried out in different geographical areas characterized by different farming practices.

\subsection{FST and Genetic Structure}

The present study shows that Somba and Lagunaire taurines are genetically close. Borgou cattle are genetically intermediate between taurines and White Fulani cattle; which support the fact that they are resulted from crossbreeding between Zebu and West African taurine. This trend was also well illustrated by [14] between native cattle populations in Benin. Other studies have also been able to distinguish zebu from West African taurine based on polymorphisms of molecular markers [1, $25,22]$. In this study, taurine cattle breeds showed various levels of introgression by Zebu blood at the average mean of $20 \%$. This introgression of taurine cattle in Benin could be explained by changes in farming practices, including the recruitment of Fulani herdsmen to keep the herds and the introduction of zebu broodstock into taurine herds that undergo crossbreeding in order to improve the productivity of the herd. The phenomenon of transhumance of zebu from Sahelian countries in the ECOWAS region also participates. Concordant results have been reported by [21] who showed that in Burkina Faso, the degree of introgression of White Fulani cattle genes in Lobi taurine was $24.3 \%$ and that of Gudali in N'dama taurine was $11 \%$. Inverse cases have been mentioned by [24], who reported levels of introgression of African taurine genes in zebu blood of $7.5 \%, 15.5 \%$, $8.1 \%, 8.5 \%, 38.2 \%$ respectively in White Fulani, Red Bororo, Sokoto Gudali, Wadara, Adamawa Gudali. All these results appeal to all actors and give rise to reflections especially for the development of a national strategy and action plan for the sustainable management of animal genetic resources in Benin with a clear definition of objectives.

\section{Conclusion}

There is still a high genetic diversity in Benin cattle populations, characterized by the polymorphisms at the studied microsatellite loci, the number of alleles per locus and population, the allelic richness and the distribution of allelic frequencies in the 6 cattle populations. However, some alleles of microsatellite markers appear to be more prominent in some cattle populations than others. White Fulani, Girolando, Borgou, Azawak x Lagunaire cattle populations are the most genetically heterogeneous. Somba and Lagunaire bulls are more homogeneous. Taurine are introgressed by zebu blood. However, they are clearly different from zebu. Girolando was distinct from all other cattle breeds. These results reveal the need to develop a strategy and an action plan for an efficient and sustainable management of local cattle resources in Benin for the wellbeing of pastoralists and future generations.

\section{Acknowledgements}

The authors thank the Higher Education Support Project (PAES) of the Economic Union of West African States (ECOWAS) for Funding the Milk Project entitled "Improvement of production processing and quality techniques for milk and milk products from cows of extensive and semi-intensive production systems in Benin (PAES-0805)".

\section{References}

[1] CountryStat/Benin (2015). Statistical Database. Accessed at http://countrystat.org/benouhttp://www.fao.org/economic/ess/c ountrystat/, the $02 / 09 / 2016$ at $8 \mathrm{pm}$.

[2] Youssao, A. K. I. (2015). National Program for Genetic Improvement. Project Support for Milk and Meat sectors (PAFILAV). Cotonou, Benin. 344 p.

[3] Lekeux, M. (2006). Doctoral Thesis: African Bovine Trypanosomosis: General and situation in Benin. National Veterinary School of Lyon, Claude Bernard University (Medicine-Pharmacy), Lyon, France. 99 p.

[4] Salifou, C. F. A., Dahouda, M., Ahounou, G. S., Kassa, S. K., Tougan, P. U., Farougou, S., Mensah, G. A., Salifou, S., Clinquart, A. and Youssao, A. K. I. (2012). Evaluation of carcass traits of Lagunaire, Borgou and Zebu Fulani Bulls raised on natural pasture in Benin. The Journal of Animal and Plant Sciences. 22 (4): 857-871. ISSN: 1018-7081.

[5] Kassa, S. K., Salifou, C. F. A., Dayo, G. K., Ahounou, G. S., Dotché, O. I., Issifou, T. M., Houaga, I., Kountinhouin, G. B., Mensah, G. A., Yapi-Gnaoré, V. and Youssao, A. K. I. (2016c). Milk production of White Fulani and Borgou cows in traditional breeding in Benin. Livestock Research for Rural Development. 28 (9).

[6] Kassa, K. and Moutouama, V. (2009). Productivity of the Somba cattle breed in the Department of Atacora: case of the municipality of Boukombe. License Memory Professional in Animal Health and Production. Department of Animal Health and Production, Polytechnic School of Abomey Calavi, University of Abomey-Calavi, Abomey-Calavi, Benin, 49 p. 
[7] Adanléhoussi, A., Bassowa, H., Defly, A., Djabakou, K., Adomefa, K. and Kouagou, N'T. (2003). The performance of Somba cattle breed in a peasant environment. Tropicultura. 21 (3): $135-141$.

[8] Kassa, S. K. (2011). Assessment of body composition and quality of carcass of Borgou and White Fulani cattle reared in the breeding system traditional. Master thesis in Biotechnology and Polygastric Management. Department of Animal Health and Production, Polytechnic School of Abomey-Calavi, University of Abomey-Calavi, AbomeyCalavi, Benin, 82 p.

[9] Kassa, S. K. (2017). Assessment of non genetic factors influencing milk production and genetic characterisation of catlle breeds in Benin. Thesis of Doctorat in Zootechnic and Genetic Improvement of Animal Productions. University of Abomey-Calavi, Abomey-Calavi, Benin. 274 p.

[10] Kassa, S. K., Ahounou, G. S., Dayo, G. K., Salifou, C. F. A., Dotché, O. I., Issifou, T. M., Kountinhouin, G. B., Mensah, G. A., Yapi-Gnaore, V. and Youssao, A. K. I. (2016a). Assessment and modeling of cow milk production Girolando, Borgou, Lagunaire and crossed Azawak x Lagunaire, bred in the semi-improved system at Benin. Journal of Applied Biosciences. 103: 9829-9840. ISSN 1997-5902.

[11] Assani, S. A., Assogba, B., Toukourou, Y. and Alkoiret, I. T. (2015). Productivity of Gudali cattle farms located in the commons of Malancity and Karimama extreme North of Benin. Livestock Research for Rural Development. 27 (7): 19.

[12] Cirad (2002). Memento of the agronomist. Ministry of Foreign Affairs, France. INRA Editions, ISBN 2-86844-1297. $1692 \mathrm{p}$.

[13] Marichatou, H., Gouro, A. S. and Kanwe, A. B. (2005). Dairy production of the breed Gudali and growth of young purebreds in the peri-urban area of Bobo-Dioulasso (Burkina Faso). Cahiers d'Agriculture. 14 (3): 291-296.

[14] Moazami-Goudarzi, K., Belemsaga, D. M. A., Ceriotti, G., Laloë, D., Fagbohoun, F., Kouagou, N'T., Sidibé, I., Codjia, V., Crimella, M. C., Grosclaude, F. and Touré, S. M. (2001). Characterization of Somba cattle breed using molecular markers. Review of Livestock and Veterinary Medicine of the Tropics. 54 (2): 129-138.

[15] Dayo, G. K., Thevenon, S., Berthier, D., Moazami-Goudarzi, K., Denis, C., Cuny, G., Eggen, A. and Gautier, M. (2009). Detection of selection signatures within candidate regions underlying trypanotolerance in outbred cattle populations. Molecular Ecology. 18: 1801-1813.
[16] Goudet, J. (2001). FSTAT. A program to estimate and test gene diversities and fixation indices (version 2.9.3). Available on: http://www2.unil.ch/popgen/softwares/fstat.htm.

[17] Foulley, J. L. and Ollivier, L. (2006). Genetic Diversity and Allelic Wealth: Concepts and application to cattle breeds. Meeting around Research on Ruminants. 13: 227-230.

[18] Belkhir, K., Borsa, P., Chikhi, L., Raufaste, N. and Bonhomme, F. (2004). GENETIX 4.05. Windows TM software for population genetics. Laboratory Genome. Populations, Interactions. CNRS UMR 5000. University of Montpellier II, Montpellier, France. Available on http://www.genetix.univmontp2.fr/genetix/genetix.htm.

[19] Rodgers, J. L. (1999). The Bootstrap. The Jackknife and the Randomization Test. A Sampling Taxonomy. Multivariate Behavioral Research. 34 (4): 441-456. DOI:10.1207/ S15327906MBR3404_2.

[20] Hubisz, M., Falush, D., Stephens, M. and Pritchard, J. (2009). Inferring weak population structure with the assistance of sample group information. Molecular Ecology Resources. 9 (5): 1322-1332. Doi: 10.1111 / j.1755-0998.2009.02591.x.

[21] Alvarez, A., Traore, A., Fernandez, I., Cuervo, M., Lecomte, T., Soudré, A., Kaboré, A., Tamboura, H. and Goyache F. (2014). Assessing introgression of Sahelian zebu genes into native Bos taurus breeds in Burkina Faso. Molecular Biology and Replication. 41: 3745-3754.

[22] Yéo, M. I. (2014). Study of the genetic diversity of a crossborder taurine race: the Baoulé taurine from southern Burkina Faso and northern Côte d'Ivoire. Memory of Master in Genetics. Specialty: Improvement of Animal Species. University of Felix HOUPHOUËT Boigny, Abidjan, Ivory Coast. 64 p.

[23] Koudandé, O. D., Dossou Gbété, G., Mujibi, F., Kibogo, H., Mburu, D., Mensah, G. A., Hanotte, O. and Van Arendonk, J. A. M. (2009). Genetic diversity and zebu genes introgression in cattle population along the coastal region of the bight of Benin. Animal Genetic Resources Information. 44: 45-55.

[24] Ibeagha-Awemu, E. M., Jann, O. C., Weimann, C. and Erhardt, G. (2004). Genetic Diversity, introgression and relationships among West/Central African cattle breeds. Genetic Selection Evolution. 36: 673-690.

[25] Yayè, A. H. (2011). Application of polymorphism of microsatellite markers for the study of the genetic diversity of Niger zebus. Memory for obtaining the Diploma of Thorough Studies (D. E. A) of Genetics and Reproduction. Institute of Rural Development, Polytechnic University of BoboDioulasso, Bobo-Dioulasso, Burkina Faso. 87 p. 